\title{
Anti-Cytokine Therapy
}

National Cancer Institute

\section{Source}

National Cancer Institute. Anti-Cytokine Therapy. NCI Thesaurus. Code C15544.

Drug treatment to reduce the activity of cytokines, particulary to control inflammation in disease modalities. 